Article

\title{
Highly Stretchable and Air-Stable PEDOT:PSS/lonic Liquid Composites for Efficient Organic Thermoelectrics
}

Seyoung Kee, Hyunho Kim, Sri Harish Kumar Paleti, Abdulrahman El Labban, Marios Neophytou, Abdul-Hamid Emwas, Husam N. Alshareef, and Derya Baran

Chem. Mater., Just Accepted Manuscript • DOI: 10.1021/acs.chemmater.9b00819 • Publication Date (Web): 09 Apr 2019

Downloaded from http://pubs.acs.org on April 15, 2019

\section{Just Accepted}

"Just Accepted" manuscripts have been peer-reviewed and accepted for publication. They are posted online prior to technical editing, formatting for publication and author proofing. The American Chemical Society provides "Just Accepted" as a service to the research community to expedite the dissemination of scientific material as soon as possible after acceptance. "Just Accepted" manuscripts appear in full in PDF format accompanied by an HTML abstract. "Just Accepted" manuscripts have been fully peer reviewed, but should not be considered the official version of record. They are citable by the Digital Object Identifier (DOI®). "Just Accepted" is an optional service offered to authors. Therefore, the "Just Accepted" Web site may not include all articles that will be published in the journal. After a manuscript is technically edited and formatted, it will be removed from the "Just Accepted" Web site and published as an ASAP article. Note that technical editing may introduce minor changes to the manuscript text and/or graphics which could affect content, and all legal disclaimers and ethical guidelines that apply to the journal pertain. ACS cannot be held responsible for errors or consequences arising from the use of information contained in these "Just Accepted" manuscripts. 


\title{
Highly Stretchable and Air-Stable
}

\section{PEDOT:PSS/Ionic Liquid Composites for}

\section{Efficient Organic Thermoelectrics}

Seyoung Kee ${ }^{\dagger}$, Hyunho Kim ${ }^{\ddagger}$, Sri Harish Kumar Paleti ${ }^{\dagger}$, Abdulrahman El Labban ${ }^{\dagger}$, Marios Neophytou ${ }^{\dagger}$ Abdul-Hamid Emwas ${ }^{\S}$, Husam N. Alshareef ${ }^{\ddagger}$ Derya Baran ${ }^{\dagger *}$

†Physical Science and Engineering Division, KAUST Solar Center (KSC), tMaterials Science and Engineering, ${ }^{\S}$ KAUST Core Labs, King Abdullah University of Science and Technology (KAUST), Thuwal 23955-6900, Saudi Arabia

\begin{abstract}
Thermoelectric (TE) generators that are capable of providing sustainable energy conversion under dynamic mechanical stresses have been explored for realizing autonomous wearable electronics. However, finding extremely deformable, efficient as well as air-stable TE materials is still a major challenge. Here, we report highly stretchable and efficient organic TE materials from aqueous composites of PEDOT:PSS and ionic liquids (ILs). In this composite, ILs simultaneously enhance Seebeck coefficient and electrical conductivity of PEDOT:PSS (up to $35 \mu \mathrm{V} \mathrm{K}^{-1}$ and $538 \mathrm{~S} \mathrm{~cm}^{-1}$, respectively) by controlling its oxidation level and nanostructure. Moreover, the resulting fibrous structure with IL-assisted soft domains leads to outstanding mechanical deformability and durability, enabling that the PEDOT:PSS/IL films simply coated on elastomeric substrates maintain the TE functionality under tensile strain $(\varepsilon)$ up to $70 \%$ and repetitive stretching cycles with $30 \% \varepsilon$ without severe degradation in TE performance.
\end{abstract}


Furthermore, we also demonstrate the long-term TE stability of PEDOT:PSS/IL composites maintaining $>80 \%$ of the initial performance after 10 days in ambient conditions. Our finding provides the potential of this novel composite as a stretchable and air-stable organic TE material.

\section{INTRODUCTION}

The realization of desired ubiquitous environment has created new human-machine interfaces through unprecedented portable/wearable technologies, such as skin-attachable displays, electronic skin system, and health monitoring sensor devices, etc. ${ }^{1-4}$ This emerging era of wearable electronics will be accelerated by integrating energy-harvesting devices, including photovoltaic, triboelectric, pyro- and piezoelectric generators, leading to the advent of truly everywhere and self-powered electronic devices, so called 'autonomous electronics'. .5 Wearable thermoelectrics (TEs) are one of the most promising solutions to produce electricity in ambient environment using human body heat that can be an ideal energy source available continuously in our daily life and be suitable to operate micro-TE devices for low power applications. $^{9}, 10$ For practical demonstration of such wearable TE devices, component materials are required to possess excellent TE properties (i.e., high dimensionless figure of merit, $Z T=S^{2} \sigma T / \kappa$, where $S, \sigma, T$, and $\kappa$ are the Seebeck coefficient, electrical conductivity, absolute temperature, and thermal conductivity) and stable TE functionality against diverse motion-induced mechanical deformations, as well as operational stability under ambient conditions. ${ }^{11,12}$

The conducting polymer (CP), poly(3,4-ethylenedioxythiophene) doped with poly(4styrenesulfonate) (PEDOT:PSS) is the most attractive organic TE material because of its: i) earth abundant elements; ii) non-toxicity and biocompatibility; iii) doping stability; iv) low $\kappa$, 
and v) easy processability from aqueous media, which offers eco-friendly and low-cost fabrication using printing techniques. ${ }^{13-15}$ Moreover, it is crucial that the low TE power factor (PF, $\left.S^{2} \sigma\right)$ of pristine PEDOT:PSS, originating from the low $\sigma\left(\approx 1 \mathrm{~S} \mathrm{~cm}^{-1}\right)$ and $S(\leq 15 \mu \mathrm{V} \mathrm{K}-$ 1), can be improved by various treatment methods. ${ }^{16,17}$ The post-treatments with high dielectric constant materials (e.g., organic polar solvents, salt solution, and acids), inducing crystalline morphologies of PEDOT:PSS films by the selective removal of insulating PSS and/or phase separation between PEDOT and PSS, have been used for $\sigma$ enhancement. ${ }^{18,}{ }^{19}$ In parallel, controlling the oxidation level of PEDOT:PSS is the most general strategy to increase its $S .{ }^{9}$ In this manner, outstanding $S$ values have been obtained using strong chemical reducing agents or electrochemical reduction process, accompanying the de-doping of PEDOT:PSS. ${ }^{20-22}$ Although these approaches are powerful, they involve complicated multi-step procedures, to increase both $\sigma$ and $S$, and incompatible processes with polymeric substrates necessary for deformable application. ${ }^{23,24}$

On the other hand, CPs have been considered as potential candidates for deformable TEs due to their inherent mechanical flexibility arising from the characteristics of amorphous and entangled polymeric materials. ${ }^{25,}{ }^{26}$ Normally, PEDOT:PSS films can withstand an external strain $(\varepsilon)$ of $\approx 5 \%,{ }^{27}$ but to fully satisfy the criteria for wearable TEs, PEDOT:PSS films are required to be durable under mechanical $\varepsilon \geq 30 \%$, which covers various dynamic human motions (e.g., bending, curling, twisting, and even stretching). ${ }^{28}$ The stretchability of PEDOT:PSS has been improved by blending with soft, elastomeric polymers, including polyurethane and polyethylene glycol, etc.; however, the addition of such insulating polymers deteriorates TE characteristic of PEDOT:PSS by lowering its $\sigma^{29-31}$ Therefore, the approach to develop extremely deformable CPs providing high TE performance has been eagerly explored. 
Herein, we report PEDOT:PSS and ionic liquid (IL) composites for aqueous-processable, highly stretchable, and efficient organic TEs. In this PEDOT:PSS/IL composite, ILs modulate the oxidation level and morphological structure of PEDOT:PSS, which leads to simultaneous increases in $S$ and $\sigma$ of PEDOT:PSS and consequently enhancement in PF. Moreover, excellent deformability and mechanical robustness are imparted by the resulting PEDOT:PSS nanofibrous structure incorporating IL-assisted soft domains. As a result, the PEDOT:PSS/IL films prepared on elastic substrate via single coating process maintain TE functionality under tensile $\varepsilon$ up to $70 \%$ and repetitive stretching cycle with $30 \% \quad \varepsilon$. Furthermore, the PEDOT:PSS/IL composite films also provides long-term stability maintaining $>80 \%$ of their initial PF after 10 days under ambient conditions.

\section{RESULTS AND DISCUSSION}

2.1. Controlling doping level and Seebeck coefficient of PEDOT:PSS using ILs. The chemical structures of PEDOT:PSS and two different types of ILs are shown in Figure 1a. The employed ILs are composed of 1-ethyl-3-methylimidazolium cation with 4,5dicyanoimidazolate and dicyanamide containing nitrogen anions, denoted as EMIM DCI (green) and EMIM DCA (red), respectively. Typically, ILs are organic salts melting below 100 ${ }^{\circ} \mathrm{C}$. Their negligible volatility and high thermal stability based on strong Coulombic interaction can be valuable features for use in TE applications. ${ }^{32}$ The physical and chemical properties of ILs (e.g., viscosity, hydrophobicity, and solubility) can be tuned by modifying their molecular structure. ${ }^{33}$ The EMIM DCI and EMIM DCA salts with short alkyl chains and hydrophilic cyano groups have good water-solubility; thus, they mix with aqueous PEDOT:PSS colloids in solution state, producing homogeneous PEDOT:PSS/IL composite films via solution process. More importantly, in this composite system, EMIM cations and basic DCI/DCA anions of ILs 
have a potential to electrostatically interact with doped $\mathrm{PEDOT}^{+}$and $\mathrm{PSS}^{-}$dopants. In this regard, as shown in Figure 1a, we anticipate that the ionic interaction and exchange between PEDOT:PSS and IL could change the doping state and further $S$ of PEDOT:PSS since $S$ is directly linked with doping level. ${ }^{34}$ Above all, we intend to investigate the influence of ILs on the oxidation level and $S$ of PEDOT:PSS.

Figure $1 \mathrm{~b}$ presents the ultraviolet-visible-near-infrared (UV-Vis-NIR) absorption spectra of pristine PEDOT:PSS and PEDOT:PSS/IL composite films. PEDOT:PSS is a heavily doped $\mathrm{CP}$ with charge carrier density $(n)$ of $\approx 10^{21} \mathrm{~cm}^{-3}$, and its strong bipolaronic and weak polaronic transitions appear at the wavelength $(\lambda)$ of $\geq 1200$ and $\approx 850 \mathrm{~nm}$, respectively. ${ }^{35-37}$ The PEDOT:PSS/IL films exhibit a distinct increase in the polaronic absorption while resulting in a concomitant decrease in the bipolaronic absorption, which indicates the introduction of ILs causes a reduction in the oxidation level of PEDOT:PSS. In addition, EMIM DCA gives rise to a larger increase in polaronic transition compared to EMIM DCI, implying EMIM DCA acts as a more effective reducing agent due to the stronger basicity of secondary amine-based DCA than that of aromatic imidazole-based DCI..$^{38}$

Raman spectroscopy analysis further proves the IL-induced reduction of PEDOT (Figure 1c). The addition of ILs narrows the width of the strong peak at $1436 \mathrm{~cm}^{-1}$ assigned to the symmetric $\mathrm{C}_{\alpha}=\mathrm{C}_{\beta}$ stretching vibration of PEDOT chains and improves the normalized intensity of the peaks located at $1300-1500 \mathrm{~cm}^{-1}$ over the background signal. These are because the dedoped PEDOT segments are more active than the doped segments to the excitation $\lambda$ of 532 nm. ${ }^{39}$ These observations are also consistent with the previous systematic studies on the dedoping phenomena of PEDOT using hydrazine with varied concentration and in situ spectroelectrochemical Raman measurement. ${ }^{20,39,40}$ According to the de-doping process in PEDOT:PSS/IL composites, the $n$ of PEDOT:PSS is clearly decreased by ILs (Figure 1d), 
where the $n$ was estimated from the plasma frequency of the films (further details can be in Figure S1).

This $n$ variation induced by ILs will alter the electronic structure of PEDOT:PSS, which is confirmed by ultraviolet photoelectron spectroscopy (UPS) analysis (Figure 1e). The pristine film exhibits a work function (WF) of $4.85 \mathrm{eV}$ that is in agreement with the values (ranging from 4.8 to $5.2 \mathrm{eV}$ ) observed in previous reports. ${ }^{41,42}$ As a result of the addition of EMIM DCI and EMIM DCA, the WF decreases to 4.60 and $4.45 \mathrm{eV}$, respectively. The same tendency in WF variation is also observed in Kelvin probe measurement (Figure S2). The WF-shifting degree in PEDOT:PSS/IL composites is coincided with the reducing capability of ILs. Thus, ILs can modulate the band structure of PEDOT:PSS over broad range of WF. Moreover, the density of states near Fermi level in low binding energy region is dramatically reduced by ILs, indicating lack of $n$ caused by the de-doping process.

To understand the IL-induced chemical de-doping effect on thermoelectric properties of PEDOT:PSS, we examined the Seebeck coefficient $(S)$ of pristine PEDOT:PSS and IL-added films as a function of temperature (Figure 1f). The pristine film shows an $S$ of $15 \mu \mathrm{V} \mathrm{K}^{-1}$. By introducing EMIM DCI and EMIM DCA, the $S$ increases to 25 and $28 \mu \mathrm{V} \mathrm{K}^{-1}$ (measured near room temperature, RT), respectively. These $S$ values are linearly increased by raising temperature and reached to 29 and $35 \mu \mathrm{V} \mathrm{K}^{-1}$ at $\approx 398 \mathrm{~K}$ for EMIM DCI and DCA respectively. Notably, the higher $S$ value is obtained by EMIM DCA having higher de-doping potential compared with EMIM DCI. Consequently, the simple addition of ILs as reducing agents enables an increase in the $S$ of PEDOT:PSS, which can be tuned with reducing ability of the ILs.

2.2 IL-induced electrical conductivity enhancement in PEDOT:PSS. In general, electrical conductivity $(\sigma)$ is proportional to $n$ and inversely proportional to $S .{ }^{9}$ Interestingly, 
the incorporation of ILs not only increases the $S$ of PEDOT:PSS by decreasing the $n$, but also enhances the $\sigma$ as shown in Figure 2a. The relatively poor $\sigma$ of pristine film is remarkably increased upon the addition of ILs, and the films with EMIM DCA and EMIM DCI exhibit a $\sigma$ of 275 and $538 \mathrm{~S} \mathrm{~cm}^{-1}$ at $\mathrm{RT}$, respectively. As the temperature increases, the $\sigma$ of the PEDOT:PSS/IL films is slightly diminished, which is associated with the $S$ increase with raising temperature. The $\sigma$ enhancement in the PEDOT:PSS/IL films is solely attributed to the increase in charge carrier mobility $(\mu)$ since the $n$ is rather decreased in this case. Figure $2 \mathrm{~b}$ presents the $\mu$ of each film, calculated from the equation, $\sigma=n e \mu$. The significant $\mu$ increase more than three orders of magnitude occurs by ILs, and maximum $\mu$ of $\approx 3.6 \mathrm{~cm}^{2} \mathrm{~V}^{-1} \mathrm{~s}^{-1}$ is obtained by EMIM DCI. Such enhancement in $\mu$ should be associated with changes in the morphological and molecular structure of the films.

The transmission electron microscopy (TEM) study provides a correlation between superior charge transport and morphological characteristics of PEDOT:PSS/IL films. The pristine film with low $\mu$ exhibits a typical amorphous structure as shown in bright-field TEM image (Figure 2c). The structural change to fibrils begins to appear in EMIM DCA-added film which has much higher $\mu$. The EMIM DCI-added film, resulting in the highest $\mu$ in this study, produces more densely developed nanofibrils with percolating network. These dark-contrast fibrils mainly represent crystalline PEDOT phases because they have a higher electron density than amorphous PSS and ILs. The formation of crystalline PEDOT fibrils is related with the IL-induced conformational change of PEDOT chains from a coil-like to linear structure, as confirmed from Raman analysis (Figure S3) ${ }^{43}$ The planar conjugated backbones of PEDOT self-assemble more efficiently and thus form the crystallized fibrillar structure. ${ }^{44}$ The electron paramagnetic resonance (EPR) data (Figure S4) also support the crystalline structure of PEDOT chains. ${ }^{44,45}$ The noticeable decrease in EPR signal of EMIM DCI-added film, despite 
the increase in polarons through de-doping process, implies the polarons on PEDOT chains form polaron-polaron pairs based on enhanced interchain coupling that is required for efficient charge transport. Therefore, it is reasonable to conclude that the enhancement in $\mu$ and consequently $\sigma$ results from this structural transition induced by ILs.

As a result of the simultaneous increase in $S$ and $\sigma$, significant enhancements in power factor (PF) are achieved in PEDOT:PSS/IL composites (Figure 2d). The films with EMIM DCA and EMIM DCI exhibit a PF of 22 and $33 \mu \mathrm{W} \mathrm{m} \mathrm{m}^{-1} \mathrm{~K}^{-2}$ at around RT, respectively. Upon temperature increase, these PF values are gradually increased up to maximum values of 33 and $42 \mu \mathrm{W} \mathrm{m} \mathrm{m}^{-1} \mathrm{~K}^{-2}$ at $398 \mathrm{~K}$ for EMIM DCA and DCI respectively, which is ascribed to the increase in $S$ according to temperature increase. In addition, the PEDOT:PSS/IL films offer high PFs with unimpaired performance in this wide temperature range owing to the thermally stable $S$ and $\sigma$ (Figure $1 \mathrm{f}$ and $2 \mathrm{a})$. It is also noteworthy that these PFs are higher than that of the solutioncoated PEDOT:PSS film mixed with dimethyl sulfoxide (DMSO) as an additive (i.e., $17 \mu \mathrm{W}$ $\mathrm{m}^{-1} \mathrm{~K}^{-2}$ ) typically used to enhance the PF and, in particular, the $\sigma$ of PEDOT:PSS (Figure S5). ${ }^{46}$

\subsection{Improvement in mechanical properties of PEDOT:PSS induced by incorporating}

ILs. Figure $3 \mathrm{a}$ depicts a schematic illustration for the morphological structure of PEDOT:PSS/IL films. As mentioned earlier, the addition of ILs results in the PEDOT nanofibrous network that is typically shown in carbon nanotubes and silver nanowires used for flexible/stretchable conductive materials. ${ }^{47,} 48$ More importantly, in the composite film, ILs can act as soft domains based on their liquid characteristics. ${ }^{49-51}$ Thus, we expect that the formation of crystalline PEDOT fibrils embedded in IL-assisted domains can exhibit distinct mechanical features. To assess exactly the difference in mechanical properties between PEDOT:PSS with and without IL, we performed stress-strain experiments on each free-standing film (Figure 3b). The addition of ILs decreases the tensile strength of PEDOT:PSS from $35 \mathrm{MPa}$ to 11-13 MPa 
and improves the elongation break point three-fold. In the overall tensile strain $(\varepsilon)$ range, the EMIM DCI-added film shows lower tensile strength relative to the EMIM DCA-added film, which can be attributed to its more intense fibrillar structure. The Young's modulus of PEDOT:PSS is drastically reduced from $479 \mathrm{MPa}$ to $55-58 \mathrm{MPa}$ by the incorporation of ILs. Furthermore, we implemented a creep recovery analysis to understand rheological properties of PEDOT:PSS/IL composite (Figure 3c). It appears that EMIM DCI-added film has viscoelastic characteristics like human cartilage and soft tissue. ${ }^{52}$ Based on the improvements in mechanical properties, we identified that the PEDOT:PSS/IL composites are potential candidates for highly stretchable TE materials.

\subsection{Mechanical stability for thermoelectric properties of PEDOT:PSS/IL composite}

films. Next, we investigated mechanical stability for TE characteristics of PEDOT:PSS/IL thinfilms coated on elastic polydimethylsiloxane (PDMS) substrates and compared with that of DMSO-added PEDOT:PSS films as reference. To prepare uniform films on PDMS's hydrophobic surface from an aqueous PEDOT:PSS solution, we added a fluoro-surfactant for better wettability and adhesion, which shows no considerable effect on TE properties. Figure $4 \mathrm{~b}$, c, and d present Seebeck coefficient $(S)$, electrical conductivity $(\sigma)$ and power factor $(\mathrm{PF})$ change, respectively, as a function of applied tensile $\varepsilon$. The $\sigma$ at each $\varepsilon$ is obtained by monitoring total resistance change $\left(R / R_{0}\right)$ and subtracting resistance increase $\left(R_{\mathrm{G}} / R_{0}\right)$ induced by geometrical shape deformation (Figure S6). Whereas the IL-free film causes decrease in both $S$ and $\sigma$ at the lower $\varepsilon$ of $10-15 \%$ and thus abrupt drop in PF, the stretchable composite films exhibit relatively stable $S$ and $\sigma$ under increasing $\varepsilon$. Consequently, the EMIM DCI-added films retain $73 \%$ of their initial $\mathrm{PF}\left(35 \mu \mathrm{W} \mathrm{m} \mathrm{m}^{-1} \mathrm{~K}^{-2}\right)$ at $70 \% \varepsilon$, which compares excellently against other CPs operating at highly stretched conditions (Figure S7). ${ }^{29,53,54}$ 
We also examined cyclic durability for $S, \sigma$, and PF of stretchable PEDOT:PSS/IL films under repetitive stretch/release with various tensile $\varepsilon$ ranging between $5 \%$ and $30 \%$, with cycles repeated 10 times at each $\varepsilon$ level (Figure 4e-g). Over the whole cyclic range, the PEDOT:PSS/IL films show stable $S$ and also maintain $\approx 90 \%$ of the initial $S$ after a total of 100 cycles with a fixed $\varepsilon$ of $30 \%$ (Figure S8). Electrical properties prove more sensitive to cyclic $\varepsilon$. Under the cycles with $\varepsilon>20 \%$, the EMIM DCA-added film exhibits relatively larger decrease in $\sigma$ than the EMIM DCI-added film (for more details, see Figure S9), indicating more elastic characteristic of PEDOT:PSS/EMIM DCI. After 60 cycles with up to $30 \% \varepsilon$, the EMIM DCI-added film still can be operated with $\approx 73 \%$ of its initial PF.

2.5. Physical deformation behavior of PEDOT:PSS/IL composite films. The distinct physical deformation behavior of PEDOT:PSS/IL films elucidates their outstanding stretchability as revealed in scanning electron microscopy (SEM) (Figure S10,11). The SEM micrograph for the IL-free film, stretched and released with a $\varepsilon$ of $20 \%$, presents clear vertical cracks perpendicular to the stretching direction. These cracks represent physical discontinuity separating conducting regions and thus result in TE breakdown. At higher $\varepsilon$, thicker and denser cracks with widths of $\approx 2.5 \mu \mathrm{m}$ are generated. On the other hand, SEM micrographs of the composite films at the same scale shows no evident cracks even at $60 \% \varepsilon$, with only very slimline defects detected at high magnification. Similar defects are also observed in the SEM micrographs of the composite films cycled 100 times with $30 \% \varepsilon$. Thus, it can be concluded that the PEDOT:PSS/IL films possess excellent physical robustness and durability against external stress, which ensures their mechanically stability for stretchable TE applications.

2.6. Ambient stability of PEDOT:PSS/IL composites. For the practical application of organic TE materials, their ambient stability must be an important consideration because $\pi$ conjugated materials are usually vulnerable to oxygen, moisture, and light. ${ }^{55}$ To address this 
issue, we conducted a long-term shelf-life test for PEDOT:PSS/IL films by storing at ambient conditions without any encapsulation. Figure $5 \mathrm{~b}$, c, and d shows PF, $\sigma$, and $S$ change versus exposure time, respectively. Compared to the IL-free film, the composite films provide more stable PF with $>80 \%$ of initial PF retained after 10 days. The difference in stability of PF is mainly attributed to the $\sigma$ variation rather than the $S$ change. The $\sigma$ of the IL-free film decreases $<75 \%$ of initial values after 10 days while the composite films maintain $>94 \%$ of initial $\sigma$. It is well known that electrical deterioration of PEDOT:PSS under ambient conditions is related to its morphological destruction induced by water absorption. ${ }^{56}$ Thus we can assume that the crystallized, nanofibrillar structure of PEDOT:PSS/IL is more robust to the moisture-induced morphology collapse. Also, as a result of the lower overall presence of PEDOT in PEDOT:PSS/IL (as shown in NIR region of Figure 1b), such composites prove less sensitive to the degradation typically seen in $\pi$-conjugated organic materials. Additionally, in the case of EMIM DCA-added film, the $\sigma$ initially increases, which may originate from a slight reoxidation of the film. ${ }^{57}$ Overall, the IL-free and de-doped composite films exhibits good $S$ stability, which can be due to the inherent doping stability of PEDOT:PSS and also the nonvolatility of ILs (unlike vapor doping) allowing them to be present in the film, preventing complete re-doping of PEDOT by PSS. Therefore, the $\sigma$ and $S$ stability of PEDOT:PSS/IL composites provides prolonged TE performance under ambient condition, thus being advantageous to demonstrate reliable organic TEs over time.

\section{Conclusion}

In conclusion, we propose a strategy to achieve a highly deformable and solutionprocessable organic TE materials by making aqueous composites of PEDOT:PSS and ILs. In the PEDOT:PSS/IL composite, ILs play an important role to increase both the $S$ and $\sigma$ of 
PEDOT:PSS, by manipulating the doping level and nanostructure of PEDOT:PSS via ionexchange process. Moreover, the resulting morphological structure of PEDOT:PSS nanofibrils embedded in IL-assisted soft domains affords substantial improvement in mechanical stretchability. The PEDOT:PSS/IL films simply coated on PDMS can be stretchable up to $70 \%$ $\varepsilon$ and reversibly under $30 \% \varepsilon$ without severe degradation in TE performance. In addition, we also demonstrate the outstanding ambient stability of PEDOT:PSS/IL films, envisioning their benefit for practical use in actual environment. Our finding is a significant step towards highly stretchable/wearable printed organic TEs. 
a)

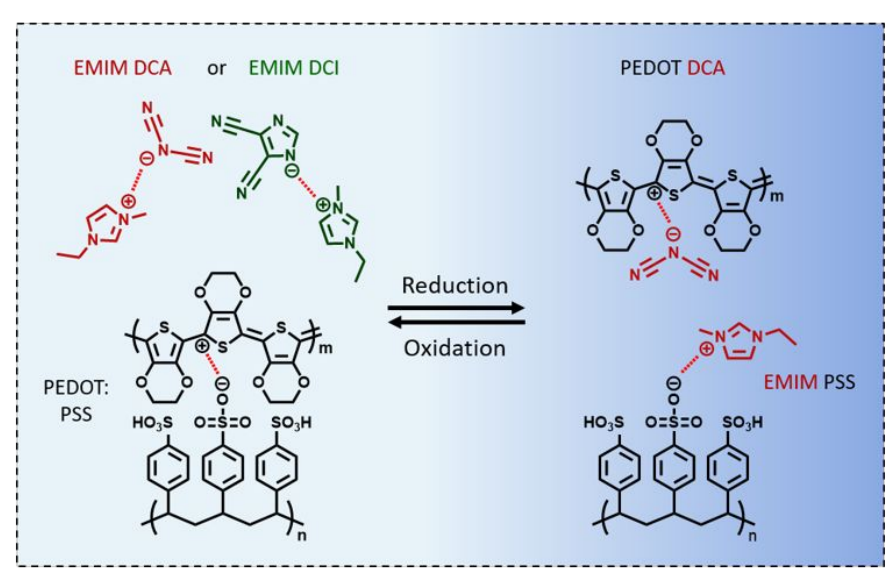

d)

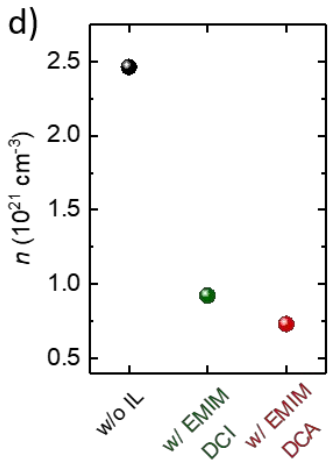

e)

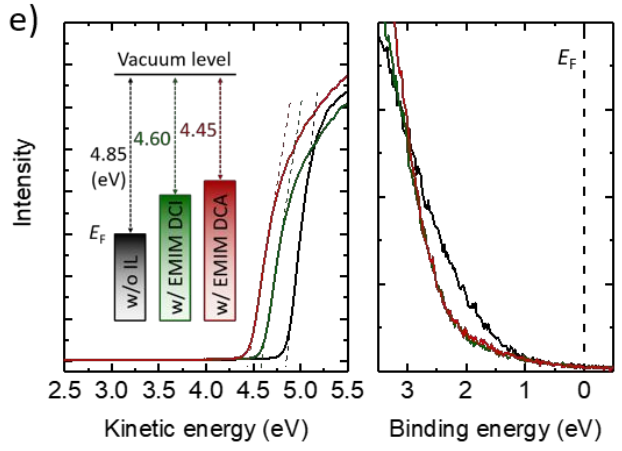

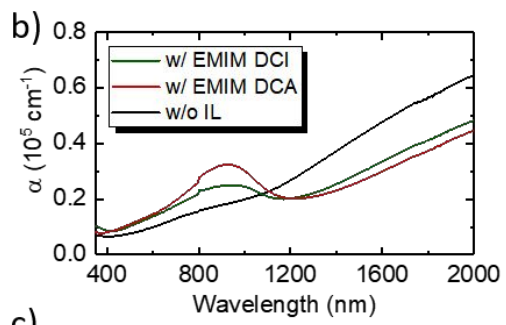

c)
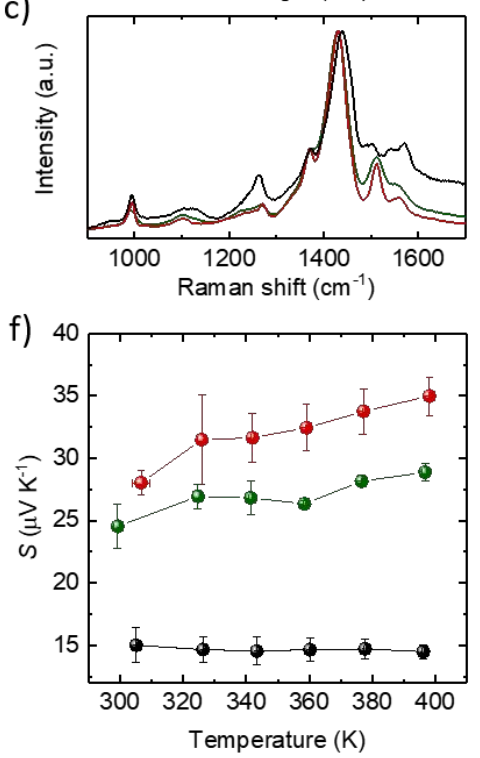

Figure 1. Controlling doping level and Seebeck coefficient of PEDOT:PSS using ILs. a) Chemical structures of PEDOT:PSS and ILs, EMIM DCI (green) and EMIM DCA (red), and a chemical reduction reaction of PEDOT:PSS through the ion exchange between PEDOT $^{+}$PSS $^{-}$ and $\mathrm{EMIM}^{+} \mathrm{DCI} / \mathrm{DCA}^{-}$. b) UV-Vis-NIR absorption coefficient spectra for pristine PEDOT:PSS film (denoted as without IL) and PEDOT:PSS films mixed with EMIM DCI and EMIM DCA (denoted as with EMIM DCI and with EMIM DCA, respectively). The thickness of the films is $\approx 150-200 \mathrm{~nm}$. c) Raman spectra of the films, obtained at excitation $\lambda$ of 532 $\mathrm{nm}$. d) Charge carrier density of the films, which is estimated from the plasma frequency in reflectance spectra for each sample. e) UPS spectra of the films at low kinetic energy region (secondary electron cut-off region) showing WF energy and at low binding energy region (near Fermi energy region). The WF of the films is obtained by linearly extrapolating the low kinetic energy onset in UPS spectra. f) Seebeck coefficient of the films as a function of temperature (ranging from approximately 298 to $398 \mathrm{~K}$ ). 

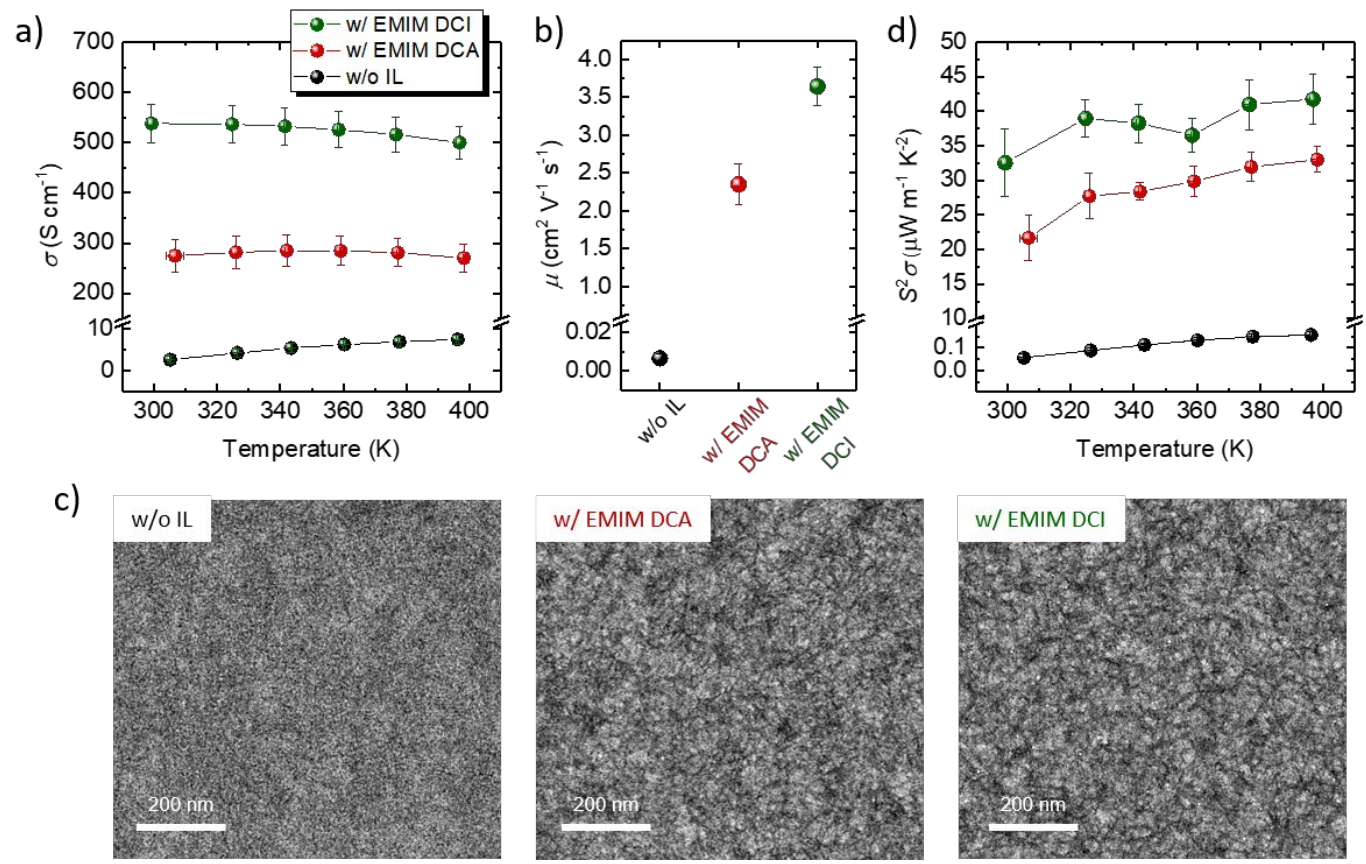

Figure 2. IL-induced electrical conductivity enhancement in PEDOT:PSS and correlation with nanostructural alteration. a) Electrical conductivity $(\sigma)$ of PEDOT:PSS films with EMIM DCI, with EMIM DCA, and without IL, as a function of temperature. b) Charge carrier mobility of the films, which is extracted from the $\sigma$ (measured at RT) based on the equation, $\sigma$ $=n e \mu,\left(\right.$ where $e$ is the electric charge, $\left.\left.\approx 1.60 \times 10^{-19} \mathrm{C}\right) . \mathrm{c}\right)$ Bright-field TEM images and $\mathrm{d}$ ) temperature-dependent power factor of the films. All scale bars are $200 \mathrm{~nm}$. 
a)

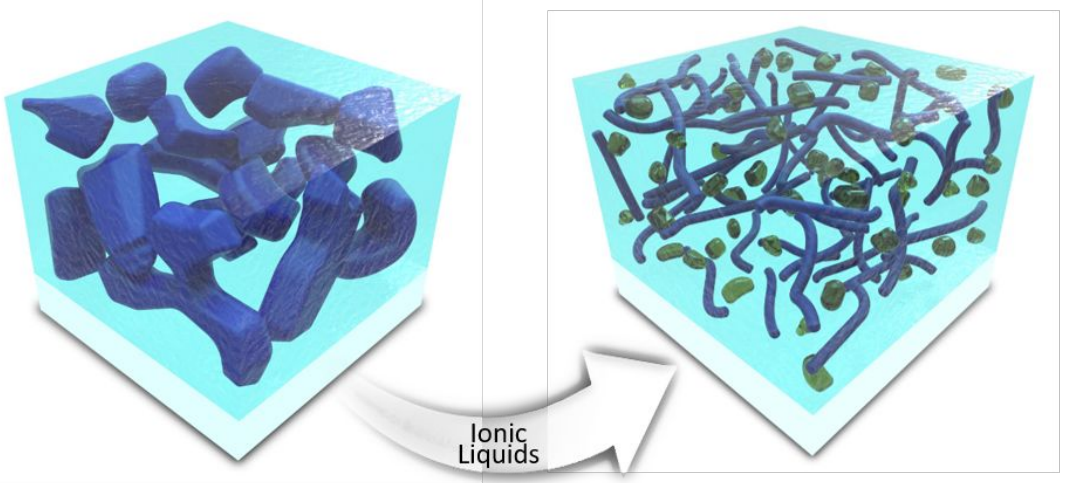

b)

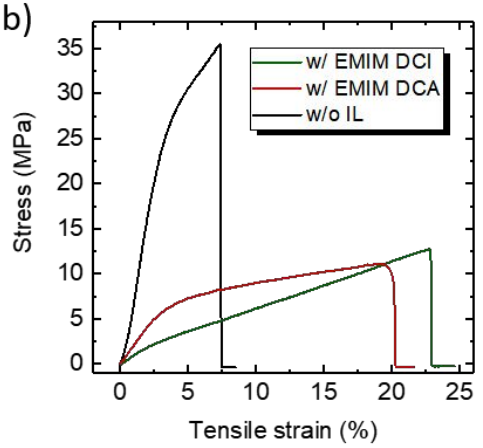

c)

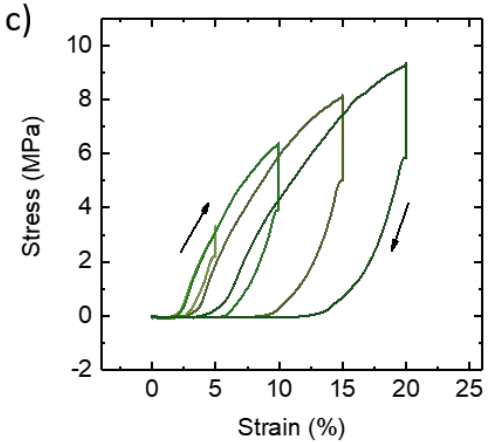

Figure 3. Improvement in mechanical properties of PEDOT:PSS induced by incorporating ILs. a) Diagram of the IL-induced morphological transition of PEDOT:PSS from amorphous structure into crystalline nanofibrils surrounded with IL-assisted soft domains. b) Stress-strain curve of free-standing PEDOT:PSS films with EMIM DCI, with EMIM DCA, and without IL. The thickness of the films is $\approx 15 \mu \mathrm{m}$. c) Creep recovery tests for PEDOT:PSS/EMIM DCI film with the strain of 5\%, 10\%, 15\%, and 20\%. 
a)
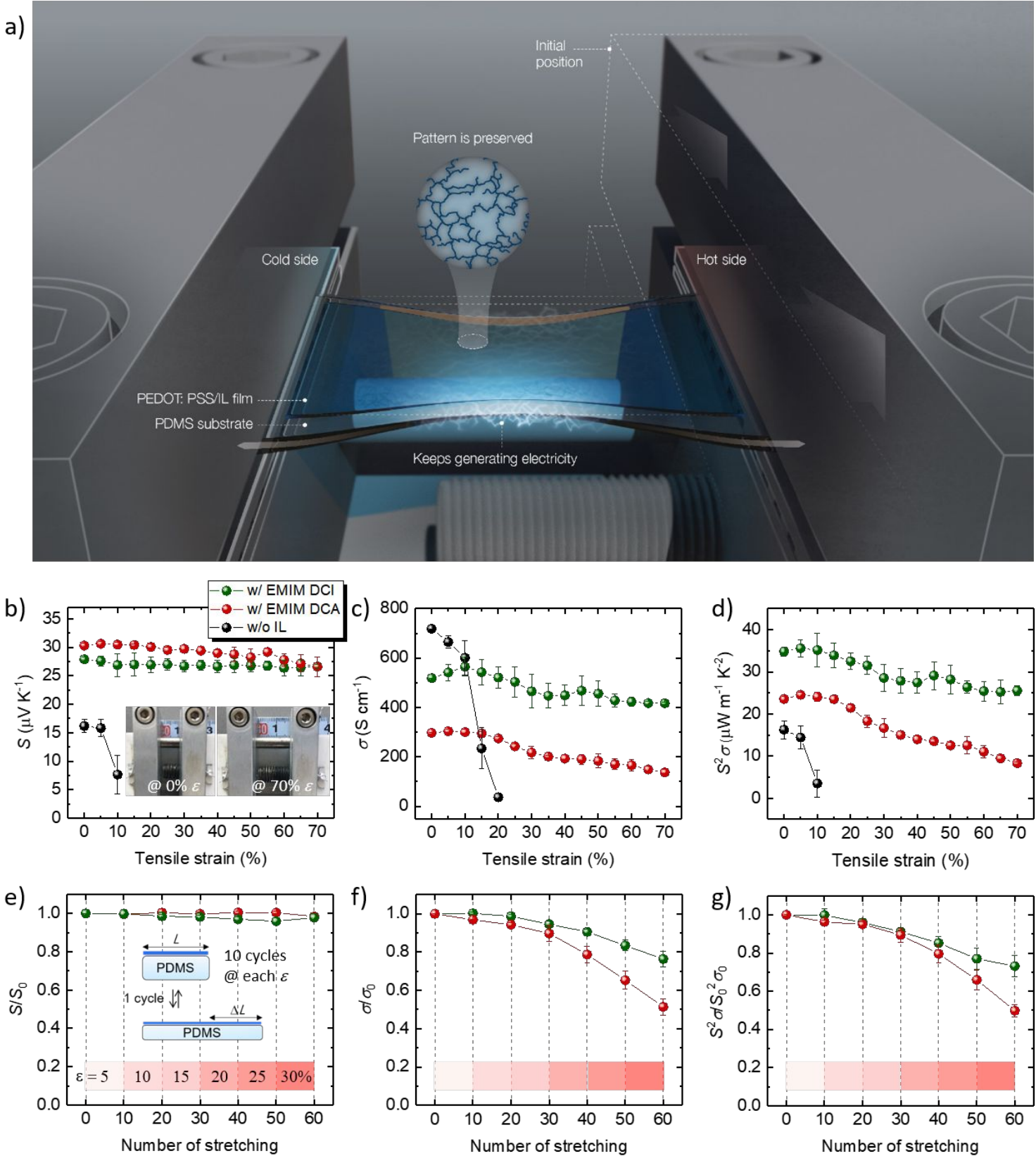

Figure 4. Mechanical stability for thermoelectric properties of PEDOT:PSS/IL composite films. a) Schematic diagram of the PEDOT:PSS/IL film loaded on the stretching instrument for thermoelectric measurements. b) Seebeck coefficient $(S)$, c) electrical conductivity $(\sigma)$, and d) power factor $(\mathrm{PF})$ change as a function of applied tensile strain $(\varepsilon)$, for PEDOT:PSS films with EMIM DCI, with EMIM DCA, and without IL (mixed with DMSO additive). The inset images in b) present the film with the $\varepsilon$ of $0 \%$ (left) and $70 \%$ (right). e) Normalized $S, \mathrm{f}$ ) $\sigma$, and g) PF of the films as a function of number of stretching. As described in the inset scheme of e), for one cycle, the film on PDMS was stretched/released with each $\varepsilon$ (elongation divided by initial length, $\Delta L / L \times 100)$. The cycles were repeated 10 times at each $\varepsilon$ stage. 
a)

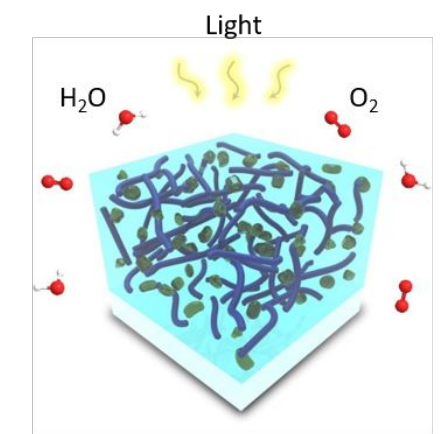

c) 12

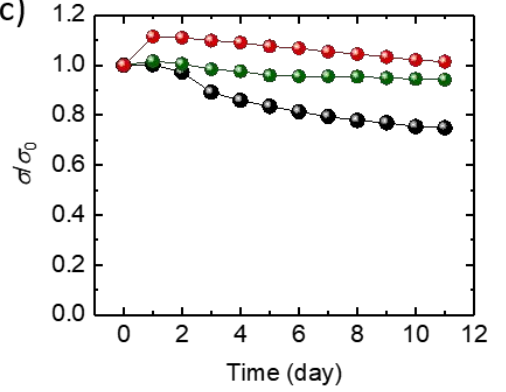

b)

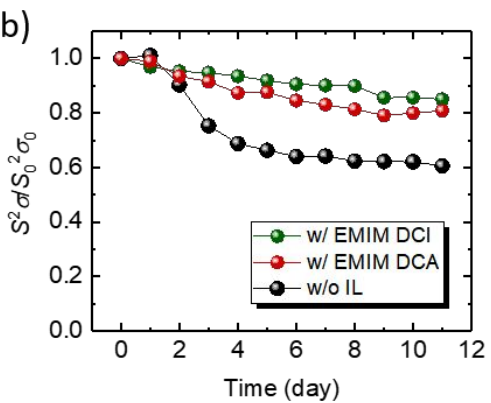

d)

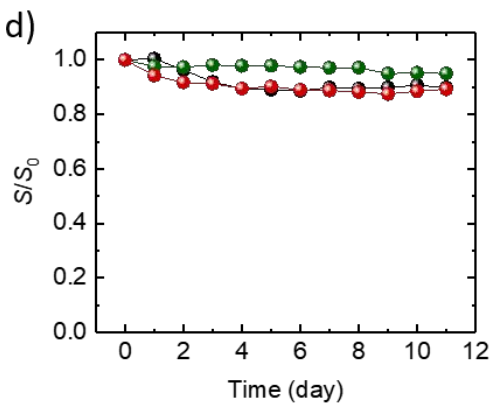

Figure 5. Ambient stability of PEDOT:PSS/IL composites. a) Schematic illustration for PEDOT:PSS/IL film exposed to oxygen, moisture, and light conditions. b)-d) Normalized power factor, electrical conductivity, and Seebeck coefficient profiles of PEDOT:PSS films with EMIM DCI, with EMIM DCA, and without IL (mixed with DMSO additive), as a function of storage time in ambient conditions without any additional encapsulation. 


\section{ASSOCIATED CONTENT}

Supporting Information. The Supporting Information is available free of charge on the ACS Publications website at DOI: XX.XXXX/acsami.XXXXXXX.

Experimental Section, reflectance spectra, Kelvin probe analysis, Raman spectra, EPR data, normalized resistance and $S$ changes data, SEM images (PDF)

\section{AUTHOR INFORMATION}

\section{Corresponding Author}

*E-mail: derya.baran@kaust.edu.sa

\section{Notes}

The authors declare no competing financial interest.

\section{ACKNOWLEDGEMENTS}

D.B. acknowledges KAUST Solar Center Competitive Fund (CCF) for financial support. Figure 4a was created by Ivan Gromicho, Scientific Illustrator at King Abdullah University of Science and Technology (KAUST).

\section{REFERENCES}

1. $\quad$ Bauer, S., Sophisticated skin. Nat. Mater. 2013, 12, 871-872.

2. Cima, M. J., Next-generation wearable electronics. Nat. Biotechnol. 2014, 32, 642-643.

3. Son, D.; Kang, J.; Vardoulis, O.; Kim, Y.; Matsuhisa, N.; Oh, J. Y.; To, J. W. F.; Mun, J.; Katsumata, T.; Liu, Y.; McGuire, A. F.; Krason, M.; Molina-Lopez, F.; Ham, J.; Kraft, U.; Lee, Y.; Yun, Y.; Tok, J. B. H.; Bao, Z., An integrated self-healable electronic skin system fabricated via dynamic reconstruction of a nanostructured conducting network. Nat. Nanotechnol. 2018, 13, 1057-1065.

4. $\quad$ Chang, H.; Kim, S.; Jin, S.; Lee, S.-W.; Yang, G.-T.; Lee, K.-Y.; Yi, H., Ultrasensitive and Highly Stable Resistive Pressure Sensors with Biomaterial-Incorporated Interfacial Layers for Wearable Health-Monitoring and Human-Machine Interfaces. ACS Appl. Mater. Interfaces 2018, 10, 1067-1076.

5. Zi, Y.; Lin, L.; Wang, J.; Wang, S.; Chen, J.; Fan, X.; Yang, P.-K.; Yi, F.; Wang, Z. L., Triboelectric-Pyroelectric-Piezoelectric Hybrid Cell for High-Efficiency EnergyHarvesting and Self-Powered Sensing. Adv. Mater. 2015, 27, 2340-2347.

6. $\quad$ Wen, Z.; Yeh, M.-H.; Guo, H.; Wang, J.; Zi, Y.; Xu, W.; Deng, J.; Zhu, L.; Wang, X.; $\mathrm{Hu}, \mathrm{C}$.; Zhu, L.; Sun, X.; Wang, Z. L., Self-powered textile for wearable electronics 
by hybridizing fiber-shaped nanogenerators, solar cells, and supercapacitors. Sci. $A d v$. 2016, 2, e1600097.

7. $\quad \mathrm{Xu}, \mathrm{S}$.; Hansen, B. J.; Wang, Z. L., Piezoelectric-nanowire-enabled power source for driving wireless microelectronics. Nat. Commun. 2010, 1, 93.

8. Park, J. W.; Park, S. J.; Kwon, O. S.; Lee, C.; Jang, J.; Jang, J.; Park, J. W.; Park, S. J.; Lee, C.; Kwon, O. S., In Situ Synthesis of Graphene/Polyselenophene Nanohybrid Materials as Highly Flexible Energy Storage Electrodes. Chem. Mater. 2014, 26, 23542360.

9. Bubnova, O.; Crispin, X., Towards polymer-based organic thermoelectric generators. Energy Environ. Sci. 2012, 5, 9345-9362.

10. Snyder, G. J.; Lim, J. R.; Huang, C.-K.; Fleurial, J.-P., Thermoelectric microdevice fabricated by a MEMS-like electrochemical process. Nat. Mater. 2003, 2, 528-531.

11. Russ, B.; Glaudell, A.; Urban, J. J.; Chabinyc, M. L.; Segalman, R. A., Organic thermoelectric materials for energy harvesting and temperature control. Nat. Rev. Mater. 2016, 1, 16050.

12. Oh, J. Y.; Lee, J. H.; Han, S. W.; Chae, S. S.; Bae, E. J.; Kang, Y. H.; Choi, W. J.; Cho, S. Y.; Lee, J.-O.; Baik, H. K.; Lee, T. I., Chemically exfoliated transition metal dichalcogenide nanosheet-based wearable thermoelectric generators. Energy Environ. Sci. 2016, 9, 1696-1705.

13. Elschner, A.; Kirchmeyer, S.; Lövenich, W.; Merker, U.; Reuter, K., PEDOT: Principles and Applications of an Intrinsically Conductive Polymer; CRC Press: Boca Raton, FL, 2011.

14. Liu, J.; Wang, X.; Li, D.; Coates, N. E.; Segalman, R. A.; Cahill, D. G., Thermal Conductivity and Elastic Constants of PEDOT:PSS with High Electrical Conductivity. Macromolecules 2015, 48, 585-591.

15. Weathers, A.; Khan, Z. U.; Brooke, R.; Evans, D.; Pettes, M. T.; Andreasen, J. W.; Crispin, X.; Shi, L., Significant Electronic Thermal Transport in the Conducting Polymer Poly(3,4-ethylenedioxythiophene). Adv. Mater. 2015, 27, 2101-2106.

16. Chen, G.; Xu, W.; Zhu, D., Recent advances in organic polymer thermoelectric composites. J. Mater. Chem. C 2017, 5, 4350-4360.

17. Yao, H.; Fan, Z.; Cheng, H.; Guan, X.; Wang, C.; Sun, K.; Ouyang, J., Recent Development of Thermoelectric Polymers and Composites. Macromol. Rapid Commun. 2018, 39, 1700727.

18. Shi, H.; Liu, C.; Jiang, Q.; Xu, J., Effective Approaches to Improve the Electrical Conductivity of PEDOT:PSS: A Review. Adv. Electron. Mater. 2015, 1, 1500017.

19. Döbbelin, M.; Marcilla, R.; Salsamendi, M.; Pozo-Gonzalo, C.; Carrasco, P. M.; Pomposo, J. A.; Mecerreyes, D., Influence of Ionic Liquids on the Electrical Conductivity and Morphology of PEDOT:PSS Films. Chem. Mater. 2007, 19, $2147-$ 2149.

20. Lee, S. H.; Park, H.; Kim, S.; Son, W.; Cheong, I. W.; Kim, J. H., Transparent and flexible organic semiconductor nanofilms with enhanced thermoelectric efficiency. $J$. Mater. Chem. A 2014, 2, 7288-7294.

21. Fan, Z.; Li, P.; Du, D.; Ouyang, J., Significantly Enhanced Thermoelectric Properties of PEDOT:PSS Films through Sequential Post-Treatments with Common Acids and Bases. Adv. Energy Mater. 2017, 7, 1602116.

22. Bubnova, O.; Berggren, M.; Crispin, X., Tuning the Thermoelectric Properties of Conducting Polymers in an Electrochemical Transistor. J. Am. Chem. Soc. 2012, 134, 16456-16459. 
23. Meng, W.; Ge, R.; Li, Z.; Tong, J.; Liu, T.; Zhao, Q.; Xiong, S.; Jiang, F.; Mao, L.; Zhou, Y., Conductivity Enhancement of PEDOT:PSS Films via Phosphoric Acid Treatment for Flexible All-Plastic Solar Cells. ACS Appl. Mater. Interfaces 2015, 7, 14089-14094.

24. Kim, N.; Kang, H.; Lee, J.-H.; Kee, S.; Lee, S. H.; Lee, K., Highly Conductive AllPlastic Electrodes Fabricated Using a Novel Chemically Controlled Transfer-Printing Method. Adv. Mater. 2015, 27, 2317-2323.

25. Savagatrup, S.; Printz, A. D.; O’Connor, T. F.; Zaretski, A. V.; Lipomi, D. J., Molecularly Stretchable Electronics. Chem. Mater. 2014, 26, 3028-3041.

26. Kee, S.; Kim, N.; Park, B.; Kim, B. S.; Hong, S.; Lee, J.-H.; Jeong, S.; Kim, A.; Jang, S.-Y.; Lee, K., Highly Deformable and See-Through Polymer Light-Emitting Diodes with All-Conducting-Polymer Electrodes. Adv. Mater. 2018, 30, 1703437.

27. Lang, U.; Naujoks, N.; Dual, J., Mechanical characterization of PEDOT:PSS thin films. Synth. Met. 2009, 159, 473-479.

28. Kim, D.-H.; Ghaffari, R.; Lu, N.; Rogers, J. A., Flexible and Stretchable Electronics for Biointegrated Devices. Annu. Rev. Biomed. Eng. 2012, 14, 113-128.

29. Taroni, P. J.; Santagiuliana, G.; Wan, K.; Calado, P.; Qiu, M.; Zhang, H.; Pugno, N. M.; Palma, M.; Stingelin-Stutzman, N.; Heeney, M.; Fenwick, O.; Baxendale, M.; Bilotti, E., Toward Stretchable Self-Powered Sensors Based on the Thermoelectric Response of PEDOT:PSS/Polyurethane Blends. Adv. Funct. Mater. 2018, 28, 1704285.

30. Li, P.; Sun, K.; Ouyang, J., Stretchable and Conductive Polymer Films Prepared by Solution Blending. ACS Appl. Mater. Interfaces 2015, 7, 18415-18423.

31. Noh, J.-S., Highly conductive and stretchable poly(dimethylsiloxane):poly(3,4ethylenedioxythiophene):poly(styrene sulfonic acid) blends for organic interconnects. RSC Advances 2014, 4, 1857-1863.

32. Armand, M.; Endres, F.; MacFarlane, D. R.; Ohno, H.; Scrosati, B., Ionic-liquid materials for the electrochemical challenges of the future. Nat. Mater. 2009, 8, 621629.

33. Wasserscheid, P.; Welton, T., Ionic Liquids in Synthesis; Wasserscheid, P.; Welton, T. Eds.; Wiley-VCH: Weinheim, 2003.

34. Xuan, Y.; Liu, X.; Desbief, S.; Leclère, P.; Fahlman, M.; Lazzaroni, R.; Berggren, M.; Cornil, J.; Emin, D.; Crispin, X., Thermoelectric properties of conducting polymers: The case of poly(3-hexylthiophene). Phys. Rev. B 2010, 82, 115454.

35. Massonnet, N.; Carella, A.; Jaudouin, O.; Rannou, P.; Laval, G.; Celle, C.; Simonato, J.-P., Improvement of the Seebeck coefficient of PEDOT:PSS by chemical reduction combined with a novel method for its transfer using free-standing thin films. J. Mater. Chem. C 2014, 2, 1278-1283.

36. Balan, A.; Baran, D.; Sariciftci, N. S.; Toppare, L., Electrochromic device and bulk heterojunction solar cell applications of poly 4,7-bis(2,3-dihydrothieno[3,4b][1,4]dioxin-5-yl)-2-dodecyl-2H-benzo[1,2,3]triazole (PBEBT). Sol. Energy Mater. Sol. Cells 2010, 94, 1797-1802.

37. Günes, S.; Baran, D.; Günbas, G.; Özyurt, F.; Fuchsbauer, A.; Sariciftci, N. S.; Toppare, L., Photovoltaic and photophysical properties of a novel bis-3-hexylthiophene substituted quinoxaline derivative. Sol. Energy Mater. Sol. Cells 2008, 92, 1162-1169.

38. Ginsburg, D., BASICITY OF AMINES. In Concerning Amines; Ginsburg, D., Ed. Pergamon Press: Oxford, 1967; pp 2-6.

39. Culebras, M.; Gómez, C. M.; Cantarero, A., Enhanced thermoelectric performance of PEDOT with different counter-ions optimized by chemical reduction. J. Mater. Chem. A 2014, 2, 10109-10115. 
40. Garreau, S.; Duvail, J. L.; Louarn, G., Spectroelectrochemical studies of poly(3,4ethylenedioxythiophene) in aqueous medium. Synth. Met. 2001, 125, 325-329.

41. Huang, J.; Miller, P. F.; Wilson, J. S.; de Mello, A. J.; de Mello, J. C.; Bradley, D. D. C., Investigation of the Effects of Doping and Post-Deposition Treatments on the Conductivity, Morphology, and Work Function of Poly(3,4ethylenedioxythiophene)/Poly(styrene sulfonate) Films. Adv. Funct. Mater. 2005, 15, 290-296.

42. Lenze, M. R.; Kronenberg, N. M.; Würthner, F.; Meerholz, K., In-situ modification of PEDOT:PSS work function using alkyl alcohols as secondary processing solvents and their impact on merocyanine based bulk heterojunction solar cells. Org. Electron. 2015, 21, 171-176.

43. Ouyang, J.; Xu, Q.; Chu, C.-W.; Yang, Y.; Li, G.; Shinar, J., On the mechanism of conductivity enhancement in poly(3,4-ethylenedioxythiophene):poly(styrene sulfonate) film through solvent treatment. Polymer 2004, 45, 8443-8450.

44. Kee, S.; Kim, N.; Kim, B. S.; Park, S.; Jang, Y. H.; Lee, S. H.; Kim, J.; Kim, J.; Kwon, S.; Lee, K., Controlling Molecular Ordering in Aqueous Conducting Polymers Using Ionic Liquids. Adv. Mater. 2016, 28, 8625-8631.

45. Bubnova, O.; Khan, Z. U.; Wang, H.; Braun, S.; Evans, D. R.; Fabretto, M.; HojatiTalemi, P.; Dagnelund, D.; Arlin, J.-B.; Geerts, Y. H.; Desbief, S.; Breiby, D. W.; Andreasen, J. W.; Lazzaroni, R.; Chen, W. M.; Zozoulenko, I.; Fahlman, M.; Murphy, P. J.; Berggren, M.; Crispin, X., Semi-metallic polymers. Nat. Mater. 2013, 13, 190194.

46. Kim, J. Y.; Jung, J. H.; Lee, D. E.; Joo, J., Enhancement of electrical conductivity of poly(3,4-ethylenedioxythiophene)/poly(4-styrenesulfonate) by a change of solvents. Synth. Met. 2002, 126, 311-316.

47. Yamada, T.; Hayamizu, Y.; Yamamoto, Y.; Yomogida, Y.; Izadi-Najafabadi, A.; Futaba, D. N.; Hata, K., A stretchable carbon nanotube strain sensor for human-motion detection. Nat. Nanotechnol. 2011, 6, 296-301.

48. $\quad$ Liang, J.; Li, L.; Niu, X.; Yu, Z.; Pei, Q., Elastomeric polymer light-emitting devices and displays. Nat. Photonics 2013, 7, 817-824.

49. Ito, A.; Yasuda, T.; Ma, X.; Watanabe, M., Sulfonated polyimide/ionic liquid composite membranes for carbon dioxide separation. Polym. J. (Tokyo, Jpn.) 2017, 49, 671-676.

50. Teo, M. Y.; Kim, N.; Kee, S.; Kim, B. S.; Kim, G.; Hong, S.; Jung, S.; Lee, K., Highly Stretchable and Highly Conductive PEDOT:PSS/Ionic Liquid Composite Transparent Electrodes for Solution-Processed Stretchable Electronics. ACS Appl. Mater. Interfaces 2017, 9, 819-826.

51. Wang, Y.; Zhu, C.; Pfattner, R.; Yan, H.; Jin, L.; Chen, S.; Molina-Lopez, F.; Lissel, F.; Liu, J.; Rabiah, N. I.; Chen, Z.; Chung, J. W.; Linder, C.; Toney, M. F.; Murmann, B.; Bao, Z., A highly stretchable, transparent, and conductive polymer. Sci. Adv. 2017, 3, e1602076.

52. Gong, M.; Zhang, L.; Zuo, Y.; Zou, Q.; Wang, Y.; Wang, L.; Li, Y., Investigation on the interpenetrating polymer networks (ipns) of polyvinyl alcohol and poly(N-vinyl pyrrolidone) hydrogel and its in vitro bioassessment. J. Appl. Polym. Sci. 2012, 125, 2799-2806.

53. Jo, J.; Oh, I.; Jin, M.-J.; Park, J.; Son, J. S.; An, K.-S.; Yoo, J.-W., Highly stretchable organic thermoelectrics with an enhanced power factor due to extended localization length. Org. Electron. 2017, 50, 367-375. 
54. Jeong, M. H.; Sanger, A.; Kang, S. B.; Jung, Y. S.; Oh, I. S.; Yoo, J. W.; Kim, G. H.; Choi, K. J., Increasing the thermoelectric power factor of solvent-treated PEDOT:PSS thin films on PDMS by stretching. J. Mater. Chem. A 2018, 6, 15621-15629.

55. Ogawa, S., Organic Electronics Materials and Devices; Springer: Toyko, 2015.

56. Bießmann, L.; Kreuzer, L. P.; Widmann, T.; Hohn, N.; Moulin, J.-F.; MüllerBuschbaum, P., Monitoring the Swelling Behavior of PEDOT:PSS Electrodes under High Humidity Conditions. ACS Appl. Mater. Interfaces 2018, 10, 9865-9872.

57. Fabiano, S.; Braun, S.; Liu, X.; Weverberghs, E.; Gerbaux, P.; Fahlman, M.; Berggren, M.; Crispin, X., Poly(ethylene imine) Impurities Induce n-doping Reaction in Organic (Semi)Conductors. Adv. Mater. 2014, 26, 6000-6006. 


\section{Table of Contents}

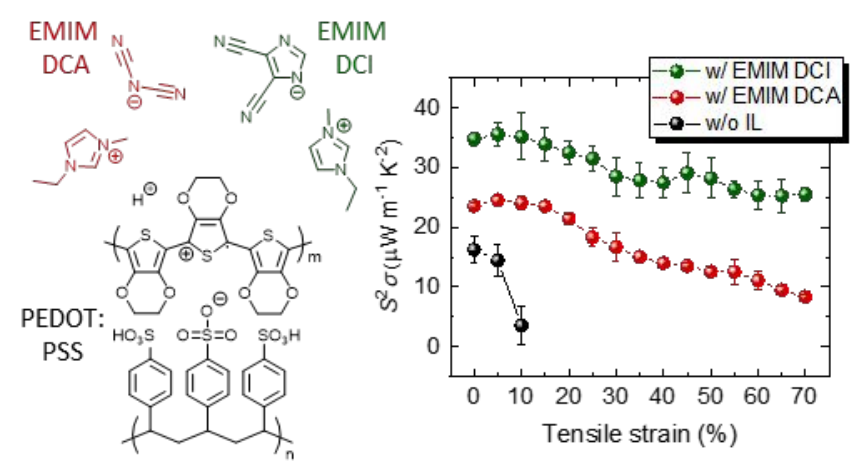

